\title{
STUDIES IN THE PHYSIOLOGY OF BLOOD VESSELS IN MAN. III. SOME EFFECTS OF RAISING AND LOWERING THE ARM UPON THE PULSE VOLUME AND BLOOD VOLUME OF THE HU- MAN FINGER TIP IN HEALTH AND IN CERTAIN DISEASES OF THE BLOOD VESSELS ${ }^{1}$
}

\author{
By ROY H. TURNER, GEORGE E. BURCH AND WILLIAM A. SODEMAN \\ (From the Department of Medicine, Tulane University of Louisiana, and the Charity Hospital \\ of Louisiana, New Orleans)
}

(Received for publication May 4, 1937)

This investigation was carried out to throw some light upon the immediate responses of nor$\mathrm{mal}$ and diseased blood vessels to changes in level of the upper extremity. Observations were limited to reactions in the finger tip, defined as that portion of the finger distal to a plane passing through the major dorsal and palmar skin creases at the terminal interphalangeal joint. While the literature abounds in qualitative studies of this nature, quantitative data are rarely encountered. Determinations were made on the effect of passive elevation and depression of the arm upon the volume of pulsations and total blood volume in the finger tip of normal subjects and those with arteriosclerosis, diastolic hypertension, arteriosclerosis and diastolic hypertension and clubbing of the fingers.

\section{METHODS}

Seventy-eight determinations were made upon 70 subjects, comprising 33 normal individuals from 12 to 65 years of age, and 37 abnormal subjects divided as follows: 13 with arteriosclerosis, 7 with diastolic hypertension, 10 with arteriosclerosis and diastolic hypertension, 2 with congenital clubbing of the fingers, 3 with acquired clubbing of the fingers, one with Raynaud's phenomenon (dead finger), and one with syphilitic aortitis with aortic regurgitation.

To eliminate the influence of menstruation and menopause, male subjects only were used. Observations were made under controlled atmospheric conditions $\left(70^{\circ} \mathrm{F}\right.$. in winter, $75^{\circ} \mathrm{F}$. in summer, relative humidity 50 per cent). After the subjects had rested in the sitting position for 30 minutes, metabolic rates were determined by the Benedict-Roth method. No dietary restrictions were made. Pulse rates and oral temperature were taken and subjects with fever eliminated. The sphygmoplethysmograph developed in our laboratory (1) was employed for the right index finger. Brachial blood pressure was

1 Aided by grants from The David Trautman Schwartz Fund, The Josiah Macy, Jr. Foundation, and The Committee on Scientific Research of the American Medical Association. determined with the mercury manometer by the auscultatory method in the opposite arm. Care was taken to eliminate constricting influences upon the circulation, the subject, however, being comfortably clothed.

The subject was seated comfortably in a chair with the right forearm resting passively in a position midway between pronation and supination upon a support as if upon the arm of a chair. The support was so constructed that movement up and down took place about a fixed point as a center which coincided with the center of movement of the shoulder joint. With motion only at the shoulder joint the arm was then raised or lowered passively to a finger tip level $45 \mathrm{~cm}$. above or below heart level. A continuous record was then made of the pulsations and other volume changes as follows : heart level, 30 seconds; lowered position, 30 seconds; heart level, 60 seconds; elevated position, 60 seconds; circulation occluded in the elevated position, 30 seconds; occlusion released and heart level, 60 seconds; lowered position, 60 seconds; and finally heart level, 60 seconds. Sudden occlusion of the circulation in the elevated position was accomplished suddenly with the use of a blood pressure cuff applied to the upper arm and connected to a 12 liter reservoir under pressure exceeding the systolic pressure of the subject by at least $50 \mathrm{~mm}$. of mercury. At this time the part studied reached its minimum volume and for the purpose of our calculations the finger tip was assumed to be bloodless. This is not strictly true. Any given volume above this base line was accepted as representing the total blood volume of the part at that stage of the observation. The volume of the bloodless soft tissue was determined by subtracting the total blood volume at heart level by a method previously described (2). Throughout these procedures efforts were made to keep psychic influences at a minimum. The records were analyzed for volume of pulsations and total change in volume of the finger tip at each level.

\section{RESULTS}

In all subjects there was a change of pulse volume with change in position. The pulse volume increased in the elevated position and decreased in the lowered position. With few exceptions, total blood volume varied in the inverse direction to the pulse volume. Table I shows the mean, 
ROY H. TURNER, GEORGE E. BURCH AND WILLIAM A. SODEMAN

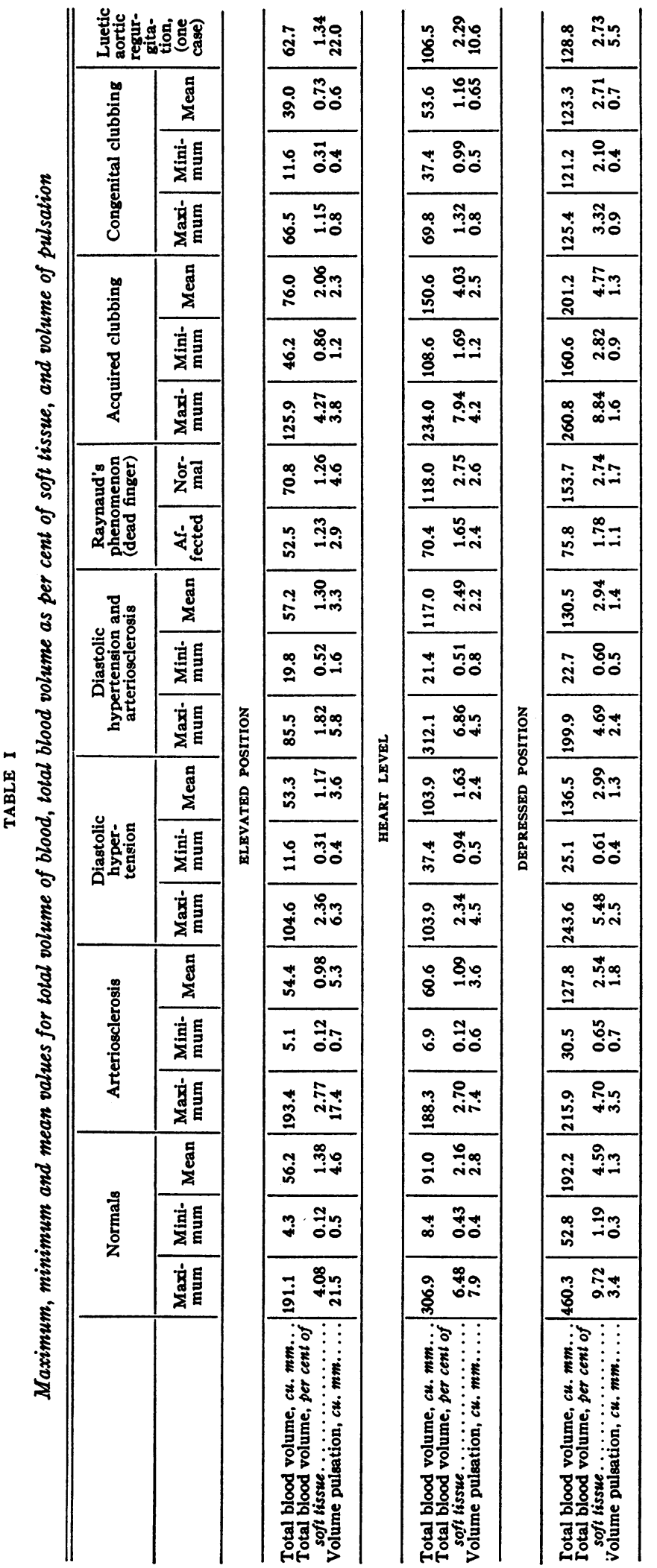


maximum and minimum values for the volume of pulsations, blood volume for the whole finger tip and as per cent of soft tissue at each level for the normal and abnormal groups.

In 39 observations on 33 normal subjects, the mean blood volumes at heart level and in the elevated and lowered positions were $90.9 \pm 6.1$, $57.6 \pm 4.4$ and $191.6 \pm 8.8 \mathrm{cu}$. $\mathrm{mm}$. respectively. The volumes of pulsation were $2.97 \pm 0.20,4.68$ \pm 0.42 and $1.30 \pm 0.08 \mathrm{cu}$. $\mathrm{mm}$. respectively. In subjects with arteriosclerosis without diastolic hypertension, the mean volume of pulsation was approximately that of the normal, and the mean total blood volume was reduced.

In subjects with diastolic hypertension, the volume of pulsations and total blood volume at heart level were reduced. In the elevated position the total blood volume was approximately that of the normal group but the volume of pulsations was again reduced. In the lowered position, the total blood volume was reduced while the volume of pulsation was that of the normal group. The range of individual values for the volume of pulsations and total blood volume was greatly limited in all positions as compared to the normal and arteriosclerotic groups.

In subjects with arteriosclerosis and diastolic hypertension the mean volume of pulsations and the total blood volume approximated closely the values obtained in subjects with diastolic hypertension alone.

In a subject with localized Raynaud's phenomenon (dead finger) with marked atrophic changes in the left index (affected) finger, distinct changes were noted at room temperature as compared to the corresponding normal (right index) finger. The involved finger showed a reduced total blood volume in all positions out of proportion to the reduction in total soft tissue volume. The volume of pulsations was slightly reduced at heart level and definitely so at the elevated and lowered positions.

In two cases of congenital clubbing of the fingers, the volume of pulsations was markedly reduced in all positions and was affected negligibly by position. The total blood volume was relatively and absolutely reduced in all positions as compared to all other groups. In patients with acquired clubbing of the fingers, values more nearly approximated normal. However, the volume of pulsations in all positions was less than in the normal while the total blood volume was greater.

One subject with syphilitic aortitis with aortic regurgitation was studied. While blood volume in all positions approximated the normal, the volume of pulsations was greater than the maximum values for the normal group.

No correlation between the observed values and pulse rate, blood pressure (systolic, diastolic or pulse pressure) or metabolic rate was found.

The results for the various groups expressed in terms of soft tissue volume indicate that the differences were not due to variations in finger size, but to actual changes in relationship of blood to tissue volume.

\section{DISCUSSION}

We have utilized in the interpretation of our data on volume change of the finger tip some observations with a recording method (3) for measuring change in light reflection from the skin of the finger pad. Its use shows that the finger tip reflects more light in the elevated and less in the depressed position than at heart level and, furthermore, furnishes sphygmograms similar in form to the volume sphygmograms. They also show similar variation in amplitude with change in position. We interpret these observations as proof that the pulse wave is manifested by volume changes in the vessels which contribute most to skin color, and that these vessels are dilated in the depressed and narrowed in the elevated position. According to Lewis (4) these vessels are principally venules and to a less extent capillaries. What is known concerning the distensibility of veins indicates that all the veins of the part share in this change in caliber. The veins leaving the finger tip have an internal pressure of more than $35 \mathrm{~mm}$. of mercury for the depressed position and only slightly above zero for the elevated position. We are unable to explain some of our observations on any other basis than that increase in total blood volume of the finger in the depressed position and diminution in the elevated position were due to change in caliber in veins and in spite of changes in the opposite direction by the arterial vessels. Any other explanation would also presume the lack of an adaptive response which func- 
tions to maintain an optimal flow through a part and optimal pressure in thin walled vessels. The predominant hemodynamic effect of diminished caliber of any given group of small vessels is increased linear velocity of flow and consequent increase in frictional resistance which diminishes pressure and volume of flow; for increased caliber the effect is the opposite. In the elevated position, with a tendency toward reduction in pressure and volume of flow, the vascular responses so change as to favor the maintenance of pressure and flow. This is accomplished mainly by arteriolar relaxation. In the depressed position, with an increase in arterial pressure, there is a response which is essentially reversed, i.e., arteriolar constriction. This tends to reduce the capillary pressure. The response, however, tends to reduce flow, the regulation of which probably becomes of secondary importance in this position. However, since friction in venous vessels in the depressed position is diminished, total resistance to flow through the finger tip is not as great as arteriolar resistance would indicate. Within limits, the regulation of total flow and of pressure in thin walled vessels can be dissociated. If arteries shared in the vascular dilatation in the depressed position, capillary and venular pressures which are, so long as flow continues, higher than pressures in the veins leaving the finger tip, would reach levels forcing rapid edema formation, which, our data indicate, does not happen. The most direct evidence we have concerning the effect of change in level upon the arterial vessels of the finger tip is derived from changes in pulse volume. We are unaware to what extent the pulse wave is manifested by volume changes in arterial vessels, but we must assume that any normal vessel must react with a volume change when subjected to the pressure pulse wave, and the pressure change during a pulse cycle is greatest in arteries. The volume pulsation of arterial vessels themselves would be directly related to diameter and inversely to their tone. For these reasons, and for others developed below, we believe that diminished tone and increased diameter of arterial vessels play chief rôles in producing increased pulse volume of the finger tip in the elevated position and that increased tone, and consequent diminished diameter, are principal fac- tors in causing the diminished pulse volume of the depressed position. Change in state of distensibility of the capillaries and venules is probably another factor. In the elevated position the minimum volume of these vessels during a pulse cycle is low, favoring a high degree of distensibility. In the depressed position, the minimum volume during the pulse cycle is high, approaching a state of maximum distension and stretch of the vessel wall. The soft tissue of the finger tip as a whole with engorgement of many vessels, no doubt, becomes a less distensible mass. The tendency for a given pressure increase to produce lessened volume increase in capillaries and venules as diameters and volume are increased is difficult to evaluate since such a change involves two factors which may vary in opposite directions. This is due to the fact that the stretching force applied within vessels depends not only upon the pressure of the liquid within the vessel but upon the surface area of the wall. As the diameter of the vessel increases, pressure remaining constant, the stretching force increases. Nevertheless, it seems most likely that in the elevated position easy distensibility of both arterial and venous vessels exists and in the depressed position just the opposite state obtains ; that is, both groups of vessels being subject to greatly increased internal pressure, the small arterial vessels react with sufficiently active increase in tone to produce diminished diameters and the small venous vessels are greatly distended probably in spite of increased active tone.

In our inquiry into factors influencing variation in pulse volume we must deal with those influences which are responsible for the tendency for the pulse wave to disappear within the vessels of the finger tip. Krogh (5) states that the difference in length of capillary paths extinguishes the pulse wave by timing the wave differently as it enters the venules by means of different capillaries. Unless the pulse wave comes to travel at extremely low velocity in these vessels, it is difficult to believe that the minute differences in their length could be the basis for a major smoothing mechanism. It seems to us that there are two principal factors, elasticity of reservoir and friction, the former probably being the chief factor in the elevated position and the latter in the de- 
pressed position. A well known mechanism for smoothing a pulsating flow employs an elastic reservoir with frictional resistance to outflow. Such an elastic reservoir tends to substitute a constant pressure head for a variable one. The resistance to outflow from the reservoir is necessary for utilization of the elastic characteristics of the reservoir. No doubt such a mechanism plays a part in the smoothing phenomenon in the finger tip. Our observations with the light reflection method show that the small veins of the finger tip exert sufficient resistance to blood flow, particularly when the diameters are diminished, as in the elevated position, to bring into play the properties of venules and capillaries as an elastic reservoir through which the blood has just passed. The magnitude of the pulse volume would indicate that this mechanism is particularly in effect in the elevated position. The other smoothing mechanism employs friction alone. The transmission of a pulse wave along a vascular channel requires the forward movement of an additional quantity of blood in order to change the pressure from diastolic to systolic. As the pulse wave enters the minute arterial vessels, the forward movement of this additional quantity of blood encounters increased frictional resistance, and the wave is subject to a greater smoothing influence than in the larger vessels. Energy expended in friction is lost in the dynamics of the circulation, while energy expended in stretching an elastic vessel wall for the pulse wave is largely restored by rebound. The smoothing effect of friction would be most marked in any given order of vessels where frictional resistance to flow was high and where the wave was manifested by a marked change in linear velocity of flow. These conditions are found in highest degree in the arterioles, and increased friction in the arterioles is probably a major factor in the diminished volume response of the finger tip to the pulse wave in the depressed position. Landis (6) has measured the pressure gradient in capillaries of the nail bed at the level of the manubrium and in the elevated and depressed positions and reported gradients which can only mean that the capillaries are, for all positions, also the site of high frictional resistance to blood flow. The opening of additional vessels with engorgement is a feature of capillary physiology which tends to prevent diminution of friction, even though the channel is also widened by increasing diameters of individual vessels. Friction which smooths the pulse wave only functions at pressures above diastolic and, of course, only in those vessels which are subject to both systolic and diastolic pressures. Our reference to friction is chiefly to friction between blood column and vessel wall. Much of this friction is expressed in the vortex phenomenon along the outer zones of the blood column. Vortex phenomena due to variations in diameter of channels and in linear velocity of flow may be an important means of energy waste and serve to smooth pulsations.

With the finger tip at heart level, diminutions in pulse volume comparable to those observed for the depressed position were noted when venous pressure was raised to $35 \mathrm{~mm}$. of mercury by use of a pressure cuff about the arm. This suggests that the mechanisms producing diminished pulse volume in the depressed position, even those involving arterial constriction, may have their origin in the pressure state of the venous vessels.

When the pulse volume of the finger tip was measured under a variety of conditions tending to produce local anoxemia, increase in pulse volume was noted, which we interpret as evidence of reactive hyperemia. Each excursion of the finger tip from heart level, as used in our routine procedure in this study, may be looked upon as a test of physiological adaptation to changed pressures, arterial and venous. As a criterion of adaptation, we have compared heart level pulse volumes immediately before and after lowering the finger tip. Normal adult subjects showed afterward increased pulse volumes 22 times, diminished volumes 14 times, and no change once, while patients with hypertension with or without arteriosclerosis showed increased volumes 6 times, diminished volumes 13 times, and no change once. The difference in response between normal and hypertensive individuals to the depressed position is capable of a number of interpretations, one of which is that it illustrates the tendency toward vasoconstriction in hypertension. This technical approach to the study of hypertension deserves further attention.

We have made an effort to resolve the principal 

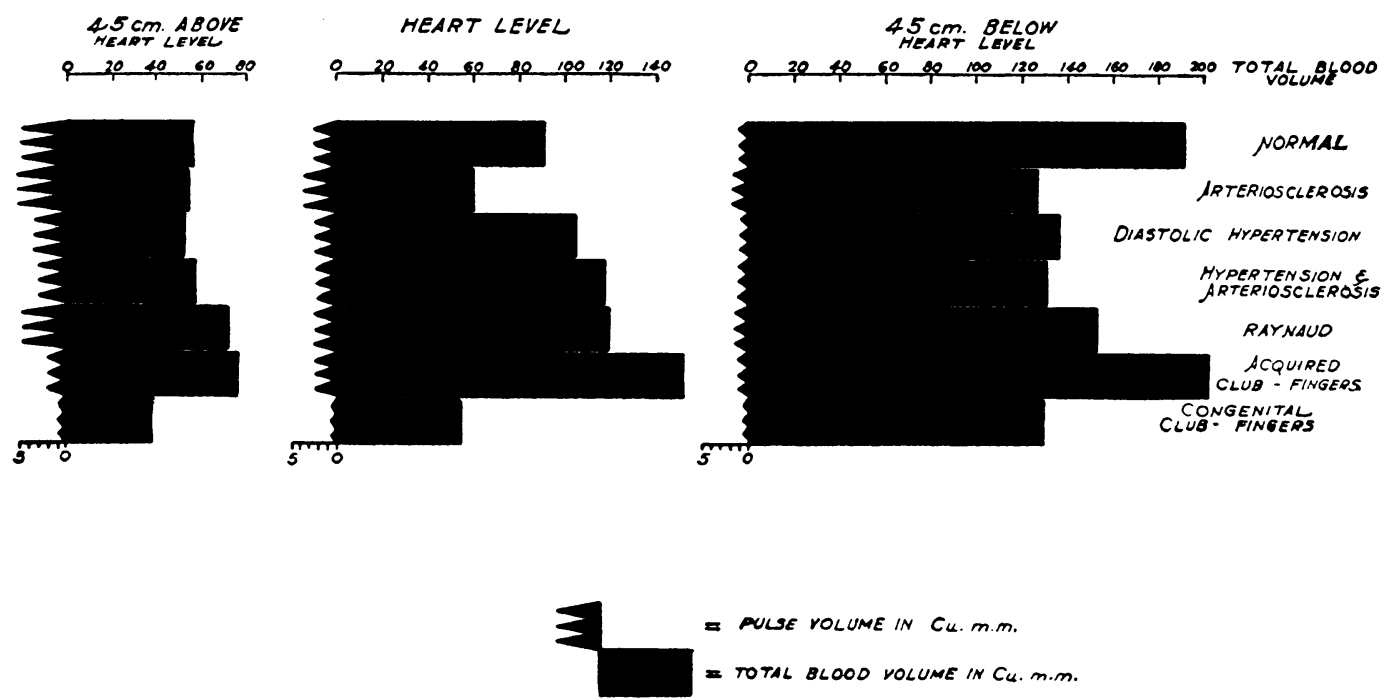

Fig. 1. Mean Values for Pulse Volume and Total Blood Volume of the Finger tip at Heart Level and at 45 cm. above and below Heart Level for Various Groups of Subjects Studied

factors involved in the expression of the volume pulse of the finger tip into quantitative values in spite of the fact that sources of error were obvious. We have used pulse pressure as representing the force producing expansion and as being unchanged by position. In our data for normal subjects there was no significant correlation between pulse pressure and pulse volume. Even in one subject, from day to day, pulse pressure and pulse volume varied in such a way as to show that they had no constant relationship. At least one other important variable seemed to be present. The ratio of pulse pressure to pulse volume gives a number which is useful in appreciating the magnitude of influences other than pulse pressure responsible for pulse volume. The volume response to a given pressure change would be modified by the number, size, length and distensibility of the vessels and other factors which would modify friction to blood flow. Changes in both size and tone of vessels most concerned with regulating pulse volume tend to give a larger volume change to a given pressure change, when the change is in the direction of less friction and vice versa. According to this mode of analysis, tone and friction are both great when the finger tip expands little in response to a given pulse pressure, and tone and friction are both low when it expands much to the same pressure. The greatest source of error probably lies in the use of pulse pressure as measured in the brachial artery and not in the arteries entering the finger tip. It seems likely that the pulse pressure in the finger tip arteries is so related to that in the brachial artery as to warrant the use of the latter in preliminary analyses based on this ratio. The factors modifying the transmission of the pressure pulse wave in larger arteries, such as the radial, are the same for the small vessels of the finger tip discussed above. Pulse pressure may diminish, that is the pulse wave may be smoothed as it passes along an artery, by either of two mechanisms involving opposite conditions, great increase in distensibility or a great increase in friction. We have observed (7) in normal arteries changes in pulse wave velocity when the hand was changed from heart level to the depressed and elevated positions indicative of increased tone of the arteries of the arm in the depressed position and diminished tone for the elevated position. Unless these changes were extreme, that is producing marked change in friction, and the changes in pulse wave velocity were not extreme, the influence of changed tone would be toward increased pulse pressure entering the depressed finger tip and diminished pulse pressure entering the elevated finger tip. In small, rigid, calcareous radial arteries the friction factor would probably predominate rather than loss in elasticity, the result 


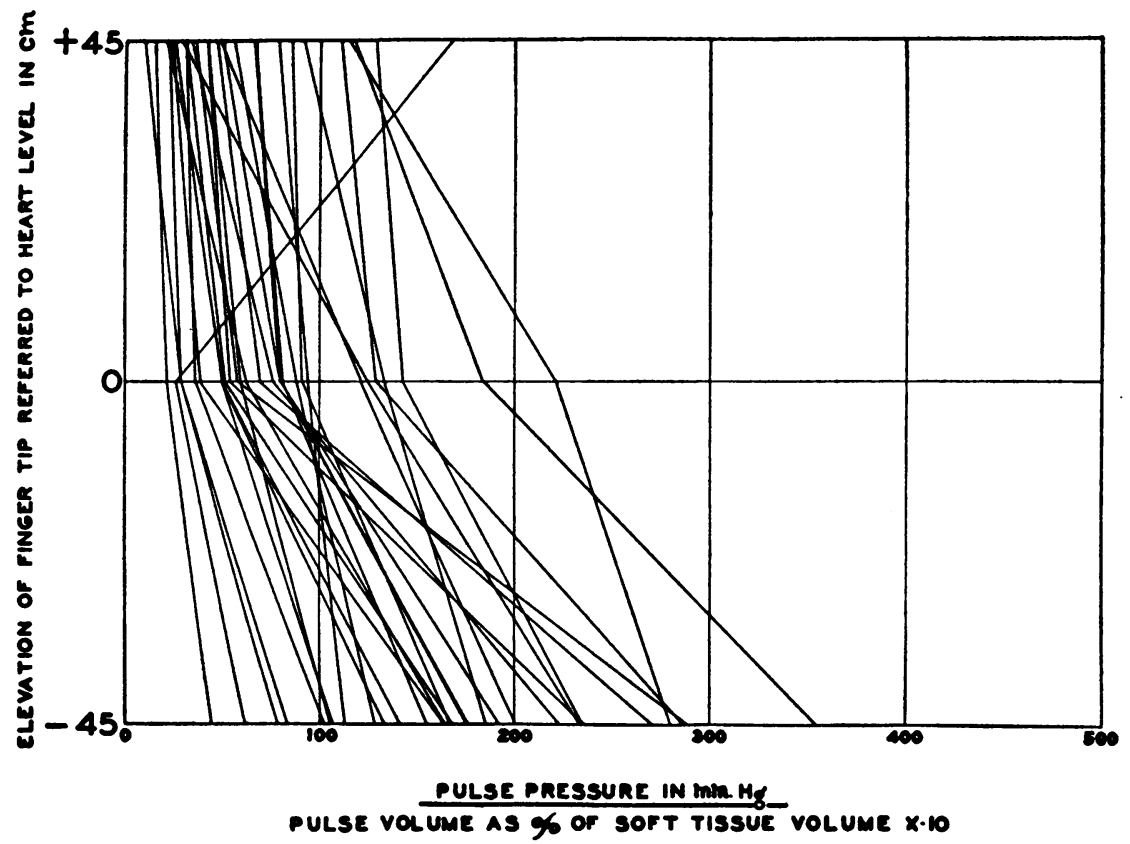

Fig. 2. Ratios for Normal Adult Subjects

Each line connects points which represent a ratio for each of three levels and based on one observation for a normal adult subject. The heavy line represents mean values for ratios.

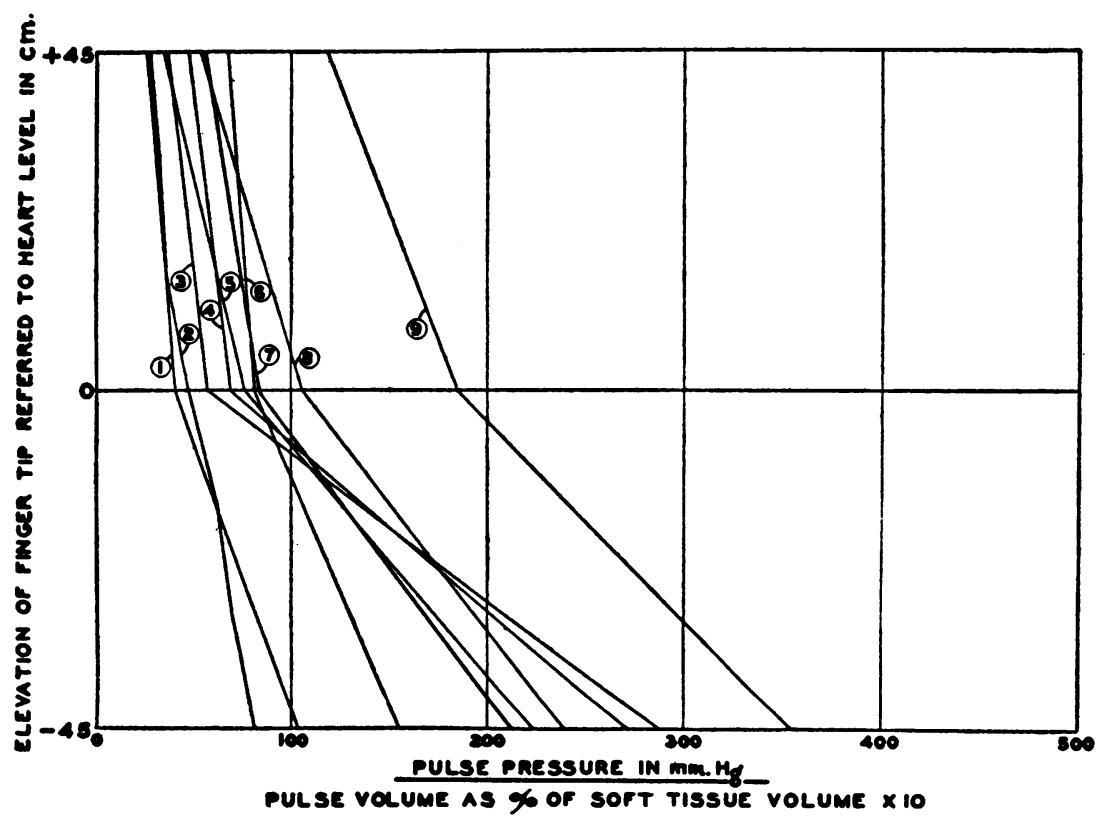

Fig. 3. Ratios Based on Repeated Observations on Different Days for the Same Normal Adult Subject

Line 2 represents ratios based on 35 measurements of pulse volume at one sitting and seven levels of the finger tip: at heart level and at 15,30 and $45 \mathrm{~cm}$. above and below heart level. Observations were made nine times at heart level, six times each at the 15 and $30 \mathrm{~cm}$. levels and three times at the extreme positions. Ratio for each level is based on mean of all measurements of pulse volume at that level. 


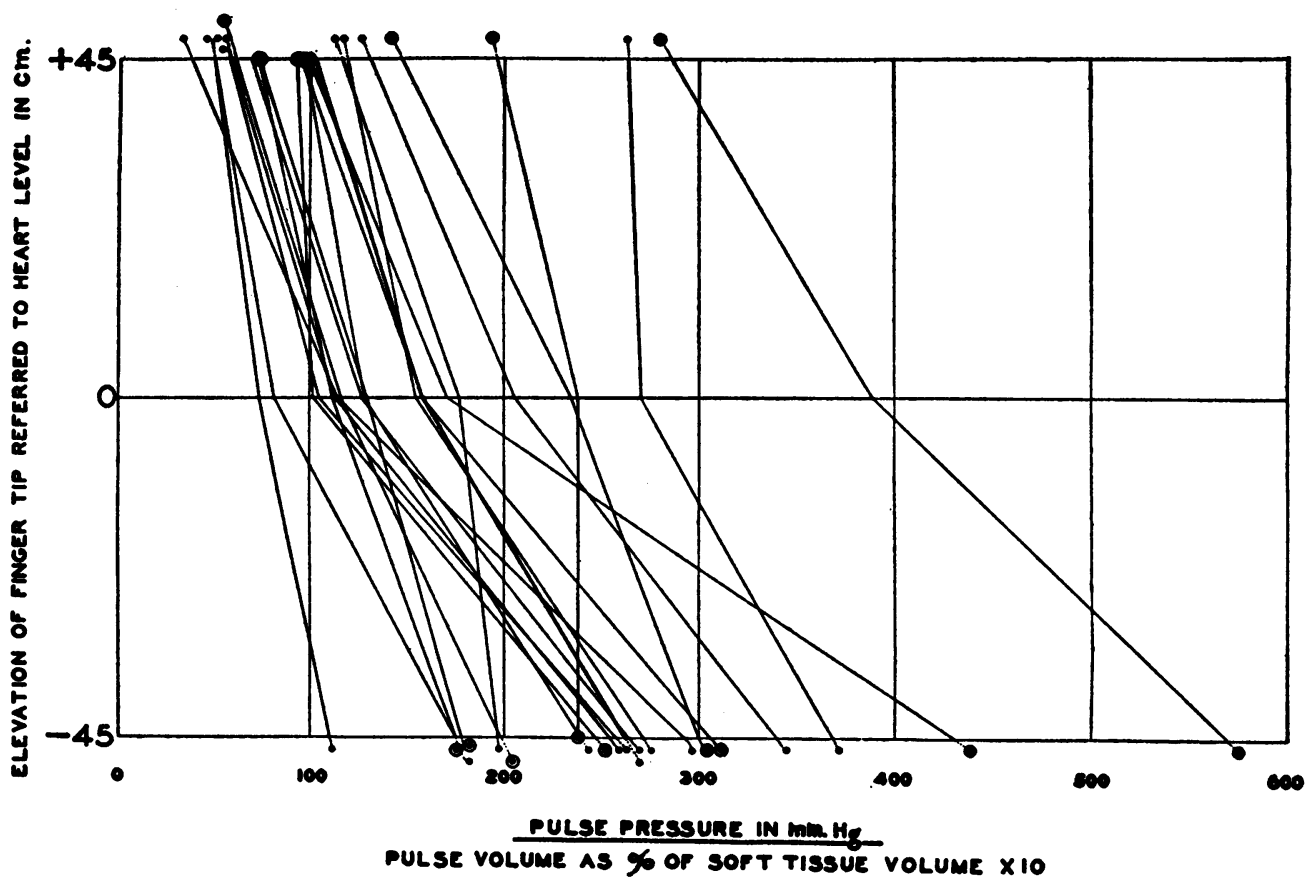

Fig. 4. Ratios for Patients with Diastolic Hypertension

Circles represent data for patients who also showed arteriosclerosis. The heavy line indicates mean values.

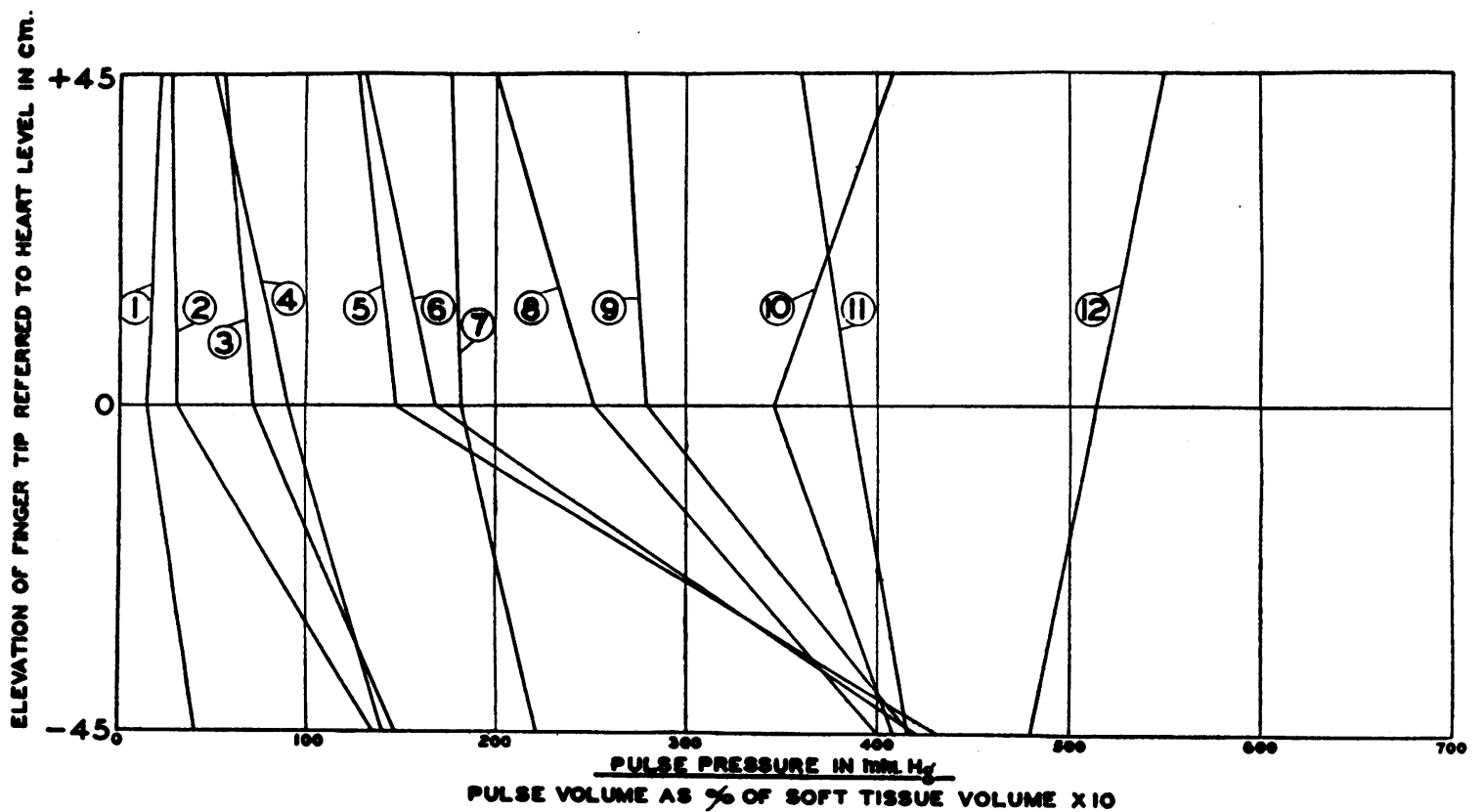

Fig. 5. Ratios for Miscellaneous Cases

(1) acquired clubbing of fingers; (2) arteriosclerosis; (3 and 4) dead finger of Raynaud's phenomenon; (5) arteriosclerosis and mild systolic hypertension; (6) anxiety neurosis; (7) clubbing of fingers of doubtful etiology ; (8) extreme arteriosclerosis of arm vessels with atrophy of finger tip; (9) arteriosclerosis; (10) hypertension and congenital clubbing of fingers; (11) extreme arteriosclerosis; (12) congenital clubbing of fingers. 
being a greatly diminished pulse pressure in the finger tip arteries.

In the graph in Figure 2 are represented the values for the ratio of pulse pressure to pulse volume for the three levels of the finger tip for the normal adult subjects, and in Figure 3 is shown the analysis of repeated studies made on one normal subject, each on a different day. Scatter is a notable feature which may be due in part to the fact that our subjects were not studied in a basal state, and in part to psychic factors. The graph indicates that tone and friction usually increased more when the change was made from heart level to the depressed position than they diminished when the change was made from heart level to the elevated position. If the graphs had been constructed so that ordinates represented changes in venous pressure, only, this difference would be largely obviated. This would seem to indicate the importance of venous pressure in the adaptive mechanisms with change in position. In the graph in Figure 4 are shown comparable data for patients with hypertension. In these patients the finger tip tended to expand less per unit of pulse pressure than for normal subjects. The calculation of ratios for most of our patients with arteriosclerosis without hypertension gave values within the range of our normal subjects. This is not surprising in view of the irregular distribution of arteriosclerosis. In Figure 5 are plotted ratios of pulse-pressure:pulse-volume for interesting cases of miscellaneous nature. One observation of a normal adolescent subject gave ratios of pulse pressure:pulse volume as high as for any of our patients with vascular disease.

Criteria employed in the selection of subjects with arteriosclerosis were purely clinical. All such patients showed marked thickness of both radial and brachial arteries. Variations in the distribution of the sclerotic process from one portion of the body to another together with the fact that clinical evidence of disease was not in the part studied make it impossible to estimate the extent of the process in the finger tip. Atrophy of the vascular bed which is a part of the pathological process in arteriosclerosis probably explains the reduction in the total blood volume observed in these subjects.

In the group with diastolic hypertension only those patients with a diastolic pressure of at least $120 \mathrm{~mm}$. of mercury on more than one occasion were studied. According to data shown in Table $\mathrm{I}$, there is a striking difference between the volume increase in the finger tip with depression between normal subjects and patients with diastolic hypertension. This difference has become less marked since observations on additional patients have been included in the analysis. For $40 \mathrm{ob}-$ servations on 33 normal subjects the mean increase in total volume with depression was 111 per cent. For 25 patients with hypertension, 9 with arteriosclerosis also, the mean increase in volume was 71 per cent. These differences are in accord with the views of Fishberg (8) that arteriolar constriction is accompanied by varying degrees of capillary and venular constriction. However, this diminished distensibility of venous vessels may be due to either anatomical or physiological peculiarity, or to both. No differences were noted between the values for total blood volume and pulse volume in patients falling into the groups of red and pale hypertension of Volhard. Volhard considers pale hypertension the result of universal vasoconstriction and red hypertension the result of " diminution of the dilatability of the large and small arteries resulting from presenile loss of muscular elements with substitution of elastic and collagenous tissue" (8).

In view of the results obtained in subjects with arteriosclerosis alone and diastolic hypertension alone, the fact that subjects with both processes approach more closely those with diastolic hypertension is not surprising.

The results obtained in the subject with Raynaud's phenomenon are explained by the relative reduction in the vascular bed.

The distinctly different results obtained in subjects with congenital clubbing and those with acquired clubbing of the fingers leads one to the possibility that the underlying mechanisms in both types are different, although the duration of the condition may explain the differences. There is no agreement as to the microscopic pathology underlying clubbing of the fingers (9). No bony changes were noted in our cases to explain these findings and we are at present unable to account for them. While our evidence is not sufficient to postulate two distinct types of clubbing of the 
fingers, the differences were striking. Further investigations to throw light upon this question are now in progress. Volume of blood in a given tissue at a given moment is of less physiological importance than is the volume of blood which passes through the tissue in a given period of time. Estimations of flow through the finger tip may be made with the same apparatus we have employed in this study by observing the volume change which follows upon sudden venous obstruction. We are at present engaged in making such studies.

\section{SUM MARY}

Changes in total blood volume and pulse volume of the finger tip due to elevation and depression $45 \mathrm{~cm}$. from heart level for a group of males including normal subjects and patients suffering from various vascular abnormalities are reported and discussed.

Pulse volume increased with elevation and decreased with depression of the finger tip and total volume changed in the opposite direction. The adaptive mechanisms are discussed in terms of behavior of various vessel groups.

The influence of position upon pulse volume is ascribed to change in distensibility of both arterial and venous vessels and to changes in frictional resistance to blood flow and consequent changes in smoothing effect on the pulse wave which are predominantly arterial.
Observations in patients with arteriosclerosis, Raynaud's phenomenon, congenital and acquired clubbing of fingers and one patient with luetic aortic regurgitation are discussed.

\section{BIBLIOGRAPHY}

1. Turner, R. H., Studies in the physiology of blood vessels in man. Apparatus and methods. I. A sensitive plethysmosphygmograph for a portion of the finger. J. Clin. Invest., 1937, 16, 777.

2. Sodeman, W. A., Studies in the physiology of blood vessels in man. Apparatus and methods. II. A method for the determination of the volume of the soft tissue about the terminal phalanx of the human finger. J. Clin. Invest., 1937, 16, 787.

3. Turner, R. H., An apparatus for recording variations in light reflection from the skin. (To be published.)

4. Lewis, T., The Blood Vessels of the Human Skin and Their Responses. Shaw and Sons, London, 1927.

5. Krogh, A., The Anatomy and Physiology of Capillaries. Yale University Press, New Haven, 1929, $2 d$ ed.

6. Landis, E., Micro-injection studies of capillary blood pressure in human skin. Heart, 1929-31, 15, 209.

7. Turner, R. H., and Sodeman, W. A., Unpublished observations.

8. Fishberg, A. M., Hypertension and Nephritis. Lea and Febiger Co., Philadelphia, 1934, 3d ed.

9. Witherspoon, J. T., Congenital and familial clubbing of the fingers and toes, with a possibly inherited tendency. Arch. Int. Med., 1936, 57, 18. 